\title{
SOME NON-ANALYTIC-HYPOELLIPTIC SUMS OF SQUARES OF VECTOR FIELDS
}

\author{
MICHAEL CHRIST
}

\begin{abstract}
Certain second-order partial differential operators, which are expressed as sums of squares of real-analytic vector fields in $\mathbb{R}^{3}$ and which are well known to be $C^{\infty}$ hypoelliptic, fail to be analytic hypoelliptic.
\end{abstract}

\section{INTRODUCTION}

A differential operator $L$ is said to be analytic hypoelliptic if whenever $u$ is a distribution such that $L u$ is real-analytic in some open set $U$, then $u$ is necessarily also real-analytic in $U$. Elliptic operators with analytic coefficients are analytic hypoelliptic, as are certain classes of subelliptic operators [GS, M2, $\mathrm{S}, \mathrm{Ta}, \mathrm{Tp}, \mathrm{Tv} 1, \mathrm{Tv} 2 \mathrm{]}$. It has been known for some time that many subelliptic operators-whose solutions are necessarily $C^{\infty}$-nonetheless fail to be analytic hypoelliptic; among the examples now known are [BG, M1, He, PR, HH, CG]. A substantial no-man's-land persists, in which neither alternative has been proved, even in rather simple cases. In this note are announced negative results for certain second-order operators. We hope that these will serve as models for larger classes of operators, rather than being mere isolated examples.

In $\mathbb{R}^{3}$ with coordinates $x, y, t$ set

$$
X=\frac{\partial}{\partial x}, \quad Y=\frac{\partial}{\partial y}-m x^{m-1} \frac{\partial}{\partial t},
$$

and

$$
L=X^{2}+Y^{2},
$$

where $m \geq 2$ is an integer. Then $L$ is hypoelliptic in the $C^{\infty}$ sense $[\mathrm{H} 1, \mathrm{~K}]$; when $m=2$, it is analytic hypoelliptic [M2, Ta, Tv2]. For $m \geq 3$ an odd integer, however, it is not analytic hypoelliptic. This was proved for $m=3$ in [He, PR], and extended to larger $m$ in [HH], but by a method which does not apply for $m$ even. In [CG] it was found that $\bar{\partial}_{b} \circ \bar{\partial}_{b}^{*}$ fails to be microlocally analytic hypoelliptic in the appropriate part of phase space, on the CR manifold $\left\{\Im\left(z_{2}\right)=\left[\Re\left(z_{1}\right)\right]^{m}\right\}$, where $m \geq 4$ is even. In appropriate coordinates for this manifold, $-\bar{\partial}_{b} \circ \bar{\partial}_{b}^{*}=(X+i Y) \circ(X-i Y)=X^{2}+Y^{2}-i[X, Y]$, where $X, Y$ are as in $(0)$.

Theorem 1. For any even integer $m \geq 4, L$ is not analytic hypoelliptic.

Despite the similarity to results just cited, this does not follow from previous methods. The proof is rooted in a phenomenon discovered for $\bar{\partial}_{b} \circ \bar{\partial}_{b}^{*}$ in [CG],

Received by the editors February 13, 1991 and, in revised form, June 10, 1991.

1991 Mathematics Subject Classification. Primary 35A20, 35B65, 35H05.

Research supported in part by grants from the National Science Foundation. 
but that argument relied heavily upon an explicit formula for the Szegö kernel $[\mathrm{N}]$, for which there appears to be no analogue in the present situation.

To place Theorem 1 in context, consider two real vector fields $X, Y$ in $\mathbb{R}^{3}$ with analytic coefficients, and suppose them to be linearly independent at each point. Say that a point $a \in \mathbb{R}^{3}$ is of type 2 if $X, Y,[X, Y]$ span the tangent space to $\mathbb{R}^{3}$ at $a$. A general result [Tv2, Ta, M2] guarantees analytic hypoellipticity at any point of type 2 , leaving open the question of what sort of degeneracy is permitted. The following conjecture has been suggested in a more general form by Trèves [Tv2]: $L=X^{2}+Y^{2}$ fails to be analytic hypoelliptic at $a$ if in any neighborhood of $a$ there exists a real curve $\gamma$, with $\gamma^{\prime}(0) \neq 0$, such that

- $\gamma(t)$ is not a point of type 2 for any $|t|<\varepsilon$, and

- $\gamma^{\prime}(t)$ belongs to the span of $X(\gamma(t)), Y(\gamma(t))$ for every $|t|<\varepsilon$.

One may hope that analytic hypoellipticity holds in all other cases. In the special case of Theorem 1, the plane $x=0$ is foliated by a one-parameter family of such curves $\gamma$.

More recently we have built on the analysis outlined below to prove that analytic hypoellipticity breaks down for $X^{2}+Y^{2}$, with $X=\partial_{x}$ and $Y=$ $\partial_{y}-b^{\prime}(x) \partial_{t}$, whenever $b$ vanishes to order exactly $m$ at some point, with $m \in\{3,4,5, \ldots\}$.

\section{OUTLINE OF PROOF}

Let $\zeta, \tau$ be variables dual to $y, t$. Taking a partial Fourier transform in these variables reduces the analysis of $L$ to that of a two-parameter family of ordinary differential operators:

$$
-\frac{d^{2}}{d x^{2}}+\left(\zeta-\tau m x^{m-1}\right)^{2}
$$

A simple change of variables reduces the general case $\tau \neq 0$ to $\tau=1$, so we set

$$
\mathscr{L}_{\zeta}=-\frac{d^{2}}{d x^{2}}+\left(\zeta-m x^{m-1}\right)^{2}
$$

It is well known [H2] that in order to prove that $L$ is not analytic hypoelliptic, it suffices to demonstrate the next result (which is already known [PR, $\mathrm{HH}]$ for odd $m$ ).

Theorem 2. Let $m \geq 3$ be an integer. Then there exist $\zeta \in \mathbb{C}$ and $f \in L^{\infty}(\mathbb{R})$, not identically equal to zero, satisfying $\mathscr{L}_{\zeta} f \equiv 0$.

For then, assuming that $\zeta$ has strictly positive imaginary part, one may set

$$
F(x, y, t)=\int_{1}^{\infty} e^{i \tau t+i \tau^{1 / m} \zeta y} f\left(\tau^{1 / m} x\right) d \tau
$$

in the region $y>0$. Then $F \in C^{\infty}$, and $L F \equiv 0$. If $f(0) \neq 0$, one calculates readily, via a change of the contour of integration, that

$$
\left|\frac{\partial^{k}}{\partial t^{k}} F(0,1,0)\right| \geq \delta^{k+1}(m k) !
$$

for some $\delta>0$. Thus $F$ is not real-analytic. If $f(0)$ does vanish, then $\frac{d}{d x} f(0) \neq 0$ and essentially the same reasoning applies to $\frac{\partial}{\partial x} \frac{\partial^{k}}{\partial t^{k}} F$. It is easy 
to check that $\mathscr{L}_{\zeta}$ has a strictly positive lowest eigenvalue for each $\zeta \in \mathbb{R}$, and that for any $\zeta$ satisfying the conclusion of Theorem $2, \bar{\zeta}$ does also; so the assumption above is legitimate.

We have only an indirect proof of the existence of (infinitely many) $\zeta$ with the property desired. Set $\gamma=-(m-1) / 2$, and $\Phi_{\zeta}(x)=\zeta x-x^{m}$. Since the coefficient of the first-order part of $\mathscr{L}_{\zeta}$ is zero, the Wronskian of any two solutions of $\mathscr{L}_{\zeta}$ is a constant function of $x$. In the next lemma we will have two such solutions for each $\zeta$, so their Wronskian will be a function of $\zeta$ alone.

Lemma 3. Let $m \geq 4$ be an even integer. For each $\zeta \in \mathbb{C}$ there exist functions $f_{\zeta}^{+}$and $f_{\zeta}^{-}$defined on $\mathbb{R}$ which satisfy $\mathscr{L}_{\zeta} f_{\zeta}^{ \pm} \equiv 0$ and

$$
\left.\left|f_{\zeta}^{ \pm}(x)-e^{\Phi_{\zeta}(x)}\right| x\right|^{\gamma} \mid=O\left(\left|e^{\Phi_{\zeta}(x)}\right| \cdot|x|^{\gamma-1}\right) \quad \text { as } x \rightarrow \pm \infty,
$$

respectively. These functions are unique, and depend holomorphically on $\zeta$. Their Wronskian, $W$, satisfies

$$
|W(\zeta)| \leq C \exp \left(C|\zeta|^{m /(m-1)}\right) \quad \forall \zeta \in \mathbb{C}
$$

for some finite $C$ and

$$
|W(\zeta)| \geq \delta \exp \left(\delta|\zeta|^{m /(m-1)}\right) \quad \forall \zeta \in \mathbb{R}
$$

for some $\delta>0$.

Now, $W$ must have at least one zero. If not, then the real part of $\log W$ would be a harmonic function on $\mathbb{C}^{1}$ with polynomial growth at infinity, hence would be a polynomial. By (2) and (3), its degree would have to be $m /(m-1)$. But for $m \geq 3, m /(m-1)$ is not an integer. ${ }^{1}$

If $W(\zeta)=0$, then $f_{\zeta}^{-}$is a constant multiple of $f_{\zeta}^{+}$. Hence both decay exponentially as $x \rightarrow \pm \infty$, therefore certainly remain bounded. Thus $f_{\zeta}^{+}$is the function sought.

The same reasoning can be made to apply for odd $m \geq 3$, with a suitable modification of (1). Further argument shows that for any $\alpha \in \mathbb{R}$, the operator $X^{2}+Y^{2}+i \alpha[X, Y]$ fails to be analytic hypoelliptic. Related results appear in $[\mathrm{C} 1, \mathrm{C} 2, \mathrm{C} 4]$.

The proof of Lemma 3 is entirely elementary; details will appear elsewhere [C3]. The existence of solutions $f_{\zeta}^{ \pm}$with the prescribed asymptotics is a special case of a standard result in the theory of ordinary differential equations with irregular singular points at infinity [CL].

\section{REFERENCES}

[BG] M. S. Baouendi and C. Goulaouic, Nonanalytic-hypoellipticity for some degenerate elliptic operators, Bull. Amer. Math. Soc. 78 (1972), 483-486.

[C1] M. Christ, Analytic hypoellipticity breaks down for weakly pseudoconvex Reinhardt domains, Internat. Math. Res. Notices 1 (1991), 31-40.

[C2] - Remarks on the breakdown of analyticity for $\partial_{b}$ and Szegö kernels, Proc. 1990 Sendai Conf. on Harmonic Analysis, Lecture Notes in Math. (to appear).

[C3] Certain sums of squares of vector fields fail to be analytic hypoelliptic, Comm. Partial Differential Equations (to appear).

${ }^{1}$ Alternatively, the Hadamard product formula guarantees that any entire function of nonintegral order has infinitely many zeros. 
[C4] On the $\bar{\partial}$ equation in weighted $L^{2}$ norms in $\mathbb{C}^{1}, \mathrm{~J}$. Geom. Anal. (to appear).

[CG] M. Christ and D. Geller, Counterexamples to analytic hypoellipticity for domains of finite type, Ann. of Math. (to appear).

[CL] E. Coddington and N. Levinson, Theory of ordinary differential equations, McGraw-Hill, New York, 1955.

[DZ] M. Derridj and C. Zuily, Régularité analytique et Gevrey pour des classes d'opérateurs elliptiques paraboliques dégénérés du second ordre, Astérisque 2, 3 (1973), 371-381.

[GS] A. Grigis and J. Sjöstrand, Front d'onde analytique et sommes de carrés de champs de vecteurs, Duke Math. J. 52 (1985), 35-51.

[HH] N. Hanges and A. A. Himonas, Singular solutions for sums of squares of vector fields, preprint.

[He] B. Helffer, Conditions nécessaires d'hypoanalyticité pour des opérateurs invariants à gauche homogènes sur un groupe nilpotent gradué, J. Differential Equations 44 (1982), 460-481.

[H1] L. Hörmander, Hypoelliptic second order differential equations, Acta Math. 119 (1967), 147-171.

[H2] - The analysis of linear partial differential operators. I, Springer-Verlag, Berlin, 1983.

[K] J. J. Kohn, Boundary behavior of $\bar{\partial}$ on weakly pseudo-convex manifolds of dimension two, J. Differential Geom. 6 (1971), 523-542.

[M1] G. Métivier, Une class d'opérateurs non hypoélliptiques analytiques, Indiana Univ. Math. J. 29 (1980), 823-860.

[M2] _ Analytic hypoellipticity for operators with multiple characteristics, Comm. Partial Differential Equations 6 (1981), 1-90.

[N] A. Nagel, Vector fields and nonisotropic metrics, Beijing Lectures in Harmonic Analysis, Ann. of Math. Stud., no. 112, Princeton Univ. Press, Princeton, NJ, 1986, pp. 241-306.

[PR] Pham The Lai and D. Robert, Sur un problème aux valeurs propres non linéaire, Israel J. Math. 36 (1980), 169-186.

[S] J. Sjöstrand, Analytic wavefront sets and operators with multiple characteristics, Hokkaido Math. J. 12 (1983), 392-433.

[Ta] D. S. Tartakoff, On the local real analyticity of solutions to $\square_{b}$ and the $\bar{\partial}$-Neumann problem, Acta Math. 145 (1980), ! 17-204.

[Tp] J.-M. Trepeau, Sur l'hypoellipticité analytique microlocale des opérateurs de type principal, Comm. Partial Differential Equations 9 (1984), 1119-1146.

[Tv1] F. Trèves, Analytic-hypoelliptic partial differential equations of principal type, Comm. Pure Appl. Math. 24 (1971), 537-570.

[Tv2] - Analytic hypo-ellipticity of a class of pseudodifferential operators with double characteristics and applications to the $\bar{\partial}$-Neumann problem, Comm. Partial Differential Equations 3 (1978), 475-642.

Department of Mathematics, University of California, Los Angeles, California 90024

E-mail address: christ@math.ucla.edu 